\title{
Patient Generated Health Data: Framework for Decision Making
}

\author{
Neetu Singh \\ Univ. of Illinois at Springfield \\ Nsing2@uis.edu
}

\author{
Upkar Varshney \\ Georgia State University \\ Uvarshney@gsu.edu
}

\author{
Sumantra Sarkar \\ State University of New York \\ Ssarkar@binghamton.edu
}

\begin{abstract}
Patient information is a major part of healthcare decision making. Although currently scattered due to multiple sources and diverse formats, decision making can be improved if the patient information is readily available in a unified manner. Mobile technologies can improve decision making by integrating patient information from multiple sources. This study explores how patient generated health data (PGHD) from multiple sources can lead to improved healthcare decision making. A semi-systematic review is conducted to analyze research articles for transparency, clarity, and complete reporting. We conceptualize the data generated by healthcare professional as primarily from EHR/EMR and the data generated by patient as primarily from mobile apps and wearables. Eight themes led to the development of Convergence Model for Patient Data (CMPD). A framework was developed to illustrate several scenarios, to identify quality and timeliness requirements in mobile healthcare environment, and to provide necessary decision support.
\end{abstract}

\section{Introduction}

Decision making for healthcare has been an important area of research due to its complexity and potential impact on patients [1]. Quality healthcare decisions [2-4] lead to improved outcomes for (a) the patient (health, quality of life, or cost), (b) the healthcare professional (workload, income, or reduced overhead and liability), and (c) hospitals, insurance companies, government, and society (cost, productivity, or healthy employees and citizens). However, presently patient information is scattered across various platforms, generated by different stakeholders. Decision making can be improved if the patient information is readily available in a unified manner. We identify the healthcare professional generated data (HPGD) as primarily from EHR/EMR and patient generated healthcare data (PGHD) [5-7] from mobile apps and wearables [8, 9].
Traditionally, healthcare decision making solely relied on HPGD retrieved from Electronic Health Record (EHR) [2, 10, 11], a central repository of all patient-related data. It has helped in quality of decision making [2], improving processes [4], and reducing errors [11]. However, HPGD, generally owned by the provider(s), is not always current, and is updated only after a patient encounter.

To improve healthcare, patients are being empowered to capture data about oneself anytime anywhere using mobile technologies (mobile apps and wearables) $[12,13]$. This could include daily information on exercise, medication adherence, wellness, sleep and activities of daily living. PGHD extends healthcare quality and coverage [3] by additional and up-to-date patient information.

Generally, healthcare decision making has been based on HPGD [2, 15], which has been used for diagnosing and treating a condition, while PGHD has been used for monitoring adherence to medication or activities [14]. We focus on how PGHD and HPGD can converge to improve healthcare decision making (Figure 1). Convergence of HPGD and PGHD can support faster decisions taken remotely and improve patient care [16-18]. Decisions taken remotely can improve the ability for quick interventions leading to better health outcomes [19]. Also, the availability of accurate and usable PGHD can improve decision making. One such scenario includes monitoring of patient's medication adherence at dose, daily, weekly, and monthly level. This can yield missing doses due to "medication holidays" and decision making can lead to suitable interventions to address such challenges.

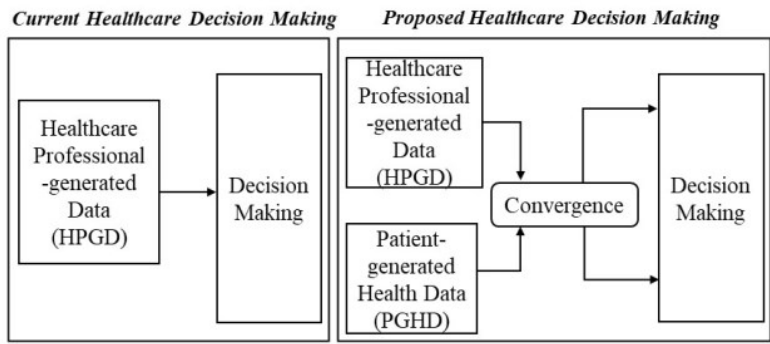

Figure 1. Healthcare decision making landscape 
However, the proposed convergence of PGHD and HPGD is complex with several challenges [16-18, 20, 21]. We present a high-level view of PGHD (mobile apps and wearables) for healthcare decision making, business models, and challenges in Figure 2.

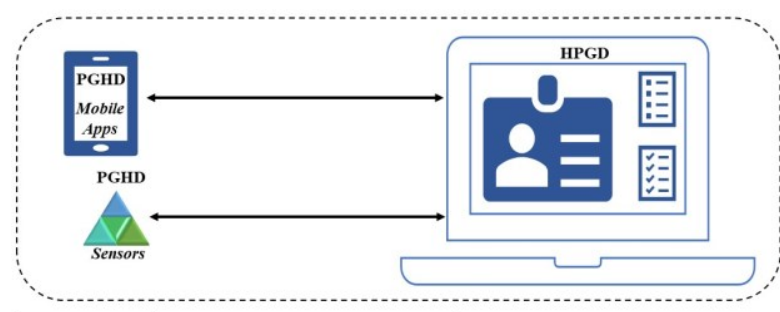

\begin{tabular}{|c|l|l|}
\hline & \multicolumn{1}{|c|}{$\begin{array}{c}\text { Patient Generated Health Data } \\
\text { (PGHD) }\end{array}$} & $\begin{array}{l}\text { Healthcare Professional Generated } \\
\text { Data (HPGD) }\end{array}$ \\
\hline Characteristics & $\begin{array}{l}\text { Unreliable } \\
\text { Novel/creative } \\
\text { Unconstrained from regulations } \\
\text { Unsecure } \\
\text { Informal decision making (patient) }\end{array}$ & $\begin{array}{l}\text { Reliable } \\
\text { Traditional } \\
\text { Constrained to regulations } \\
\text { Secure } \\
\text { Formal decision making (HP) }\end{array}$ \\
\hline $\begin{array}{c}\text { Business } \\
\text { Models }\end{array}$ & $\begin{array}{l}\text { App Specific: Involving } \\
\text { sharing/selling of patient's health } \\
\text { information to 3rd party }\end{array}$ & $\begin{array}{l}\text { EHR Specific: Not involving } \\
\text { sharing/selling of patient's health } \\
\text { information to 3rd party }\end{array}$ \\
\hline Challenges & \multicolumn{2}{|c|}{$\begin{array}{l}\text { Potential for legal problems and liabilities (Poor quality of information } \\
\text { leading to poor decisions resulting in negative outcomes for a patient.) }\end{array}$} \\
\hline
\end{tabular}

Figure 2. Schematic for Patient Generated Data for Healthcare Decision Making

In the context of convergence of PGHD and HPGD, our research questions are:

RQ1: What are the challenges in convergence of PGHD and HPGD, and how can they be addressed?

RQ2: How can health care decision making be improved by using PGHD in addition to HPGD?

Towards that goal, a semi-systematic review is used to analyze research articles for transparency, clarity, and complete reporting [22-24]. We then conducted a systematic literature review using the PRISMA (Preferred Reporting Items for Systematic Review and Meta-Analysis) approach $[25,26]$. PRISMA consists of identification, screening, eligibility and inclusion. Eight themes emerged, which we integrated towards the development of Convergence Model for Patient Data (CMPD). We then develop a framework to illustrate scenarios, to identify quality and timeliness requirements in mobile healthcare environment, and to provide necessary decision support.

\section{Methodology and literature review}

\subsection{Methodology}

The aim of the semi-systematic literature review was to gather, analyze and systematize [27] the convergence of PGHD and HPGD for healthcare decision making [1, 14, 28], following the guidelines from Snyder [29].
PGHD is a rapidly growing field which focuses on data captured via mobile apps and wearables [30, 31]. HPGD historically has been data captured in patient encounters with HP. As PGHD and HPGD terms are more recent additions to the healthcare glossary, we searched for articles using inclusive keywords in EBSCOHOST and JSTOR databases. We also searched for major informatics and IS journals (Figure 3). We then conducted a systematic literature review using the PRISMA (Preferred Reporting Items for Systematic Review and Meta-Analysis) approach [25, 26]. Figure 3 gives an overview of the process followed where the studies were identified, screened, assessed for eligibility, and were included for review. The inclusion and exclusion criteria of articles are included in each of the four stages.

\subsection{Literature review}

We used ("Mobile health" OR "Mobile app") AND ("EHR" OR "EMR") as search terms in the "Abstract" of articles in the databases. To make our search robust, we used variations of the keywords: m-health, mhealth, and EMR. Our search resulted in a total of 368 articles: 327 from EBSCOHOST $\left(\mathrm{N}_{\text {EBSCOHOST }}=327\right)$, 22 from JSTOR $\left(\mathrm{N}_{\text {JSTOR }}=22\right)$ and 19 from major informatics and IS journals $\left(\mathrm{N}_{\text {MAJOR-IS }}=19\right)$. We screened 368 articles $\left(\mathrm{N}_{\text {SCREENED }}=368\right)$ and excluded 100 (N $\mathrm{N}_{\text {EXCLUDED- }}$ SCREENED $=100)$ duplicate and redundant articles. The remaining 268 articles $\left(\mathrm{N}_{\text {ELIGIBLE }}=268\right)$ were assessed for the eligibility criterion of PRISMA. Therefore, 176 articles $\left(\mathrm{N}_{\text {EXCLUDED-ELIGIBLE }}=176\right)$ which were not fulltext were excluded. This resulted in 92 articles $\left(\mathrm{N}_{\text {INCLUDED }}=92\right)$ which were further reviewed by focusing on "mobile health" and "EHR" convergence.

Each researcher individually evaluated these articles and then the team synthesized the findings collectively. In case there was a difference of opinion concerning whether an article should be considered or not, the researchers discussed and resolved the conflict. Text data from the abstract and articles were analyzed through an iterative process following the principles of hermeneutics [32]. Themes were identified following the guidelines of qualitative analysis from Miles et al. [33]. Researchers observed that some articles simply used the terminologies "m-health," "mobile health," and/or "EHR" for references with no emphasis on the usage, implementation, and/or convergence of m-health and EHR. This led to the exclusion of additional thirtynine articles ( $\left.\mathrm{N}_{\text {EXCLUDED-FINAL }}=39\right)$. Finally, the remaining 53 articles $\left(\mathrm{N}_{\text {INCLUDED-FINAL }}=53\right)$ were examined in detail to determine how convergence of PGHD and HPGD data facilitated healthcare decision making. 


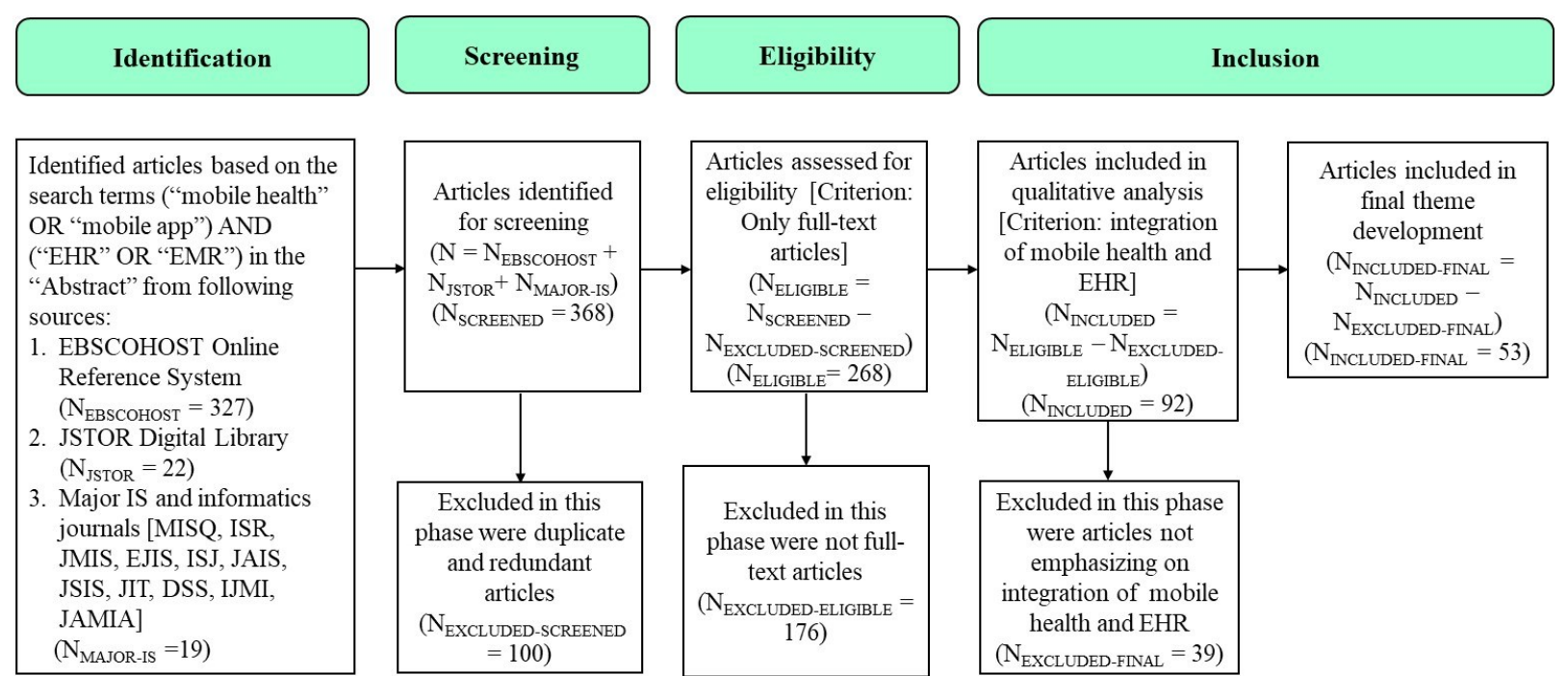

EBSCOHOST (https://www.ebsco.com/products/research-databases) is an online reference system consisting of 375 databases, including MEDLINE Complete, PUBMED, CINAHL, Business Source Complete, Dynamic Health, Health Library, Global Health, Academic Search Complete, and PsycINFO. JSTOR (https://about.jstor.org/) is a digital library of 12 million academic journal articles, books, and primary sources across 75 disciplines, including Medicine and Allied Health, Social Sciences, and Science and Mathematics. Major informatics and IS journals are JAMIA, IJMI, MISQ, ISR, JMIS, EJIS, ISJ, JAIS, JSIS, JIT, \& DSS.

Figure 3. Literature Review Based on PRISMA Approach

\section{Results}

\subsection{Themes from PGHD and HPGD studies}

The final list of 53 articles were examined with an emphasis on the convergence of PGHD and HPGD. Major gaps were identified, and several nonoverlapping patterns emerged. We classified the patterns into twenty intermediate themes. After several iterations, eight final themes emerged with little overlap (Figure 4). 37\% of the articles (19/53) focused on convergence, its challenges, privacy and security, and trends. $25 \%$ of the articles implemented a specific component of convergence (13/53), while $21 \%(11 / 53)$ developed prototypes. 9\% of the articles (5/53) considered user training or testing of prototypes with doctors and medical students, and the remaining 9\% articles $(5 / 53)$ focused on standardization and compliance.

3.1.1 Convergence Requirements. The articles (Table 1) considered the convergence of HPGD applications and decision support systems to improve decision making, patient care, and quality of life (QoL) [34]. Resistance to EHR adoption using mobile infrastructure [35], the use of mobile devices to integrate QoL [34] and clinical trials in EHR [36], and technologies and strategies to improve outcomes [37, 38] and related challenges have been identified. Standardized data exchange and process to integrate HPGD applications with PGHD applications in diverse settings globally are proposed. There is a need for tools, guidelines, models, frameworks, and metrics to assist in the evaluation of proposed solutions. Further, decision-making research can study the benefits of convergence at different levels and generalization of these findings in other settings.

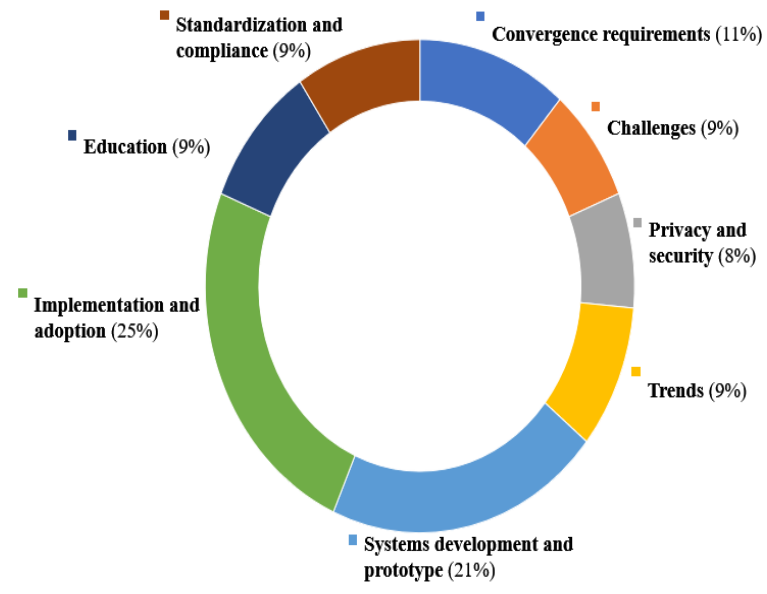

Figure 4. Themes from PGHD and HPGD studies

3.1.2 Challenges. The articles (Table 1) focused on challenges related to standardization of data generated by PGHD applications [39], implementation of health IT [40], and barriers to implementation of PGHD applications for capturing patient data [41] have been identified. The intervention of HPs for reporting data generated by mobile apps \& wearables and gaining insights from patient data have been proposed as unique solutions [41, 42]. Overload of nurses in the converged environment, privacy and security related to social media, data granularity, and the generalization of solutions can be studied. 
3.1.3 Privacy and Security. The articles (Table 1) focused on security in data sharing at different capacities, data storage in the cloud, and implementation of $\mathrm{m}$-health in regulatory compliance. The key insights include security risk associated with data sharing and PGHD application implementation [43] and implementing HIPAA in patient data accessible through cloud $[44,45]$. The challenges associated with data sharing, tradeoffs between protecting data and the advantages of convergence, and ensuring security and cloud performance can be addressed.

3.1.4 Trends. The trends (Table 1) will lead to better decision making once the challenges of mobile devices, mobile applications, and regulatory compliance in different settings are addressed. The trends identified are extended by proposing mobile device in "hospital modes" [46], identifying several $\mathrm{m}$-health applications to be reliable, accurate, and FDA-certified [47] including the evidence for various clinical informatics approaches [48].

3.1.5 Systems development and prototypes. The articles (Table 1) focused on prototypes of PGHD applications, clinical DSS, prototypes for clinicians, and applications for self-management and personalized messaging. Evaluation included simulation and user tests including beta test, pilot test and case studies. The privacy and security concerns such as secure bidirectional exchange of encrypted information $[16,18$, 21] were addressed.

3.1.6 Implementation and adoption. The articles (Table 1) focused on chronic diseases such as stroke, diabetes, and heart disease. Quality of life and end-oflife care were also studied. Some studies have implemented FHIR (Fast Health Interoperability Resources) technologies with mobile technologies [49, 50]. PGHD applications positively affected decision making and could improve productivity and quality of care. Mobile devices can support different functionalities and lead to better and more effective healthcare as compared to paper-based systems.

3.1.7 Education. The articles (Table 1) focused on educational activities for medical and pharmacy students, nurses, and other healthcare professionals [51, 52]. PGHD applications can enhance abilities to develop and employ self-regulatory and informatics skills. Simulated patient records, as an effective teaching aid, can lead to better patient outcomes [53]. The educational activities will lead to improved productivity and better outcomes [54]. Further work can include activities for patients - as individuals and as a group - to improve adherence and outcomes.

3.1.8 Standardization and compliance. The articles (Table 1) focused on access, HPGD application development, and ethics [55]. Standardization and compliance pose a difficult challenge due to the dynamic nature of technologies and regulations [56]. Management must balance among multiple factors: regulatory needs including reimbursement criteria and employee productivity to achieve desirable outcomes. A study proposed a middleware model based on HL7 to support data interoperability [57]. FDA certified PGHD applications can make healthcare professionals and HPGD application providers trust patient data can help in standardizing the data for better decision making. Further work can be done to test PGHD applications and infrastructure for compliance with reporting requirements and to identify challenges in implementing various frameworks.

Table 1. Themes and Classification of Papers

\begin{tabular}{|c|c|c|c|c|c|c|c|c|}
\hline Article & CR & CH & PS & TR & SDP & IA & ED & SC \\
\hline$[50]$ & & & & & & $\mathrm{X}$ & & \\
\hline$[16]$ & & & & & $\mathrm{X}$ & & & \\
\hline$[58]$ & & & & & & $\mathrm{X}$ & & \\
\hline$[59]$ & & & & & & $\mathrm{X}$ & & \\
\hline$[57]$ & & & & & & & & $\mathrm{X}$ \\
\hline$[60]$ & & & & & & $\mathrm{X}$ & & \\
\hline$[49]$ & & & & & & $\mathrm{X}$ & & \\
\hline$[61]$ & & & $\mathrm{X}$ & & & & & \\
\hline$[62]$ & & & & & & $\mathrm{X}$ & & \\
\hline$[37]$ & $\mathrm{X}$ & & & & & & & \\
\hline$[63]$ & & & & & $\mathrm{X}$ & & & \\
\hline$[64]$ & & & & & & $\mathrm{X}$ & & \\
\hline$[55]$ & & & & & & & & $\mathrm{X}$ \\
\hline$[65]$ & & & & & & & $\mathrm{X}$ & \\
\hline$[41]$ & & $\mathrm{X}$ & & & & & & \\
\hline$[42]$ & & $\mathrm{X}$ & & & & & & \\
\hline$[66]$ & & & & & & $\mathrm{X}$ & & \\
\hline$[67]$ & $\mathrm{X}$ & & & & & & & \\
\hline$[18]$ & & & & & $\mathrm{X}$ & & & \\
\hline$[38]$ & $\mathrm{X}$ & & & & & & & \\
\hline$[68]$ & & & & & & $\mathrm{X}$ & & \\
\hline$[69]$ & & & & & & $\mathrm{X}$ & & \\
\hline$[70]$ & & & & & $\mathrm{X}$ & & & \\
\hline$[71]$ & & & & & $\mathrm{X}$ & & & \\
\hline$[72]$ & & & & & $\mathrm{X}$ & & & \\
\hline$[73]$ & & & & & $\mathrm{X}$ & & & \\
\hline$[51]$ & & & & & & & $\mathrm{X}$ & \\
\hline$[46]$ & & & & $\mathrm{X}$ & & & & \\
\hline$[44]$ & & & $\mathrm{X}$ & & & & & \\
\hline$[40]$ & & $\mathrm{X}$ & & & & & & \\
\hline$[74]$ & & & & $\mathrm{X}$ & & & & \\
\hline$[39]$ & & $\mathrm{X}$ & & & & & & \\
\hline$[75]$ & & & & & & & & $\mathrm{X}$ \\
\hline$[52]$ & & & & & & & $\mathrm{X}$ & \\
\hline$[36]$ & $\mathrm{X}$ & & & & & & & \\
\hline$[53]$ & & & & & & & $\mathrm{X}$ & \\
\hline$[76]$ & & & & & & & & $\mathrm{X}$ \\
\hline$[77]$ & & & & & $\mathrm{X}$ & & & \\
\hline$[43]$ & & & $\mathrm{X}$ & & & & & \\
\hline$[78]$ & & & & & $\mathrm{X}$ & & & \\
\hline$[48]$ & & & & $\mathrm{X}$ & & & & \\
\hline$[56]$ & & & & & & & & $\mathrm{X}$ \\
\hline
\end{tabular}




\begin{tabular}{|l|l|l|l|l|l|l|l|l|}
\hline$[79]$ & & & & & & $\mathrm{X}$ & & \\
\hline$[45]$ & & & $\mathrm{X}$ & & & & & \\
\hline$[47]$ & & & & $\mathrm{X}$ & & & & \\
\hline$[80]$ & & & & & & $\mathrm{X}$ & & \\
\hline$[17]$ & & & & & $\mathrm{X}$ & & & \\
\hline$[81]$ & & & & $\mathrm{X}$ & & & & \\
\hline$[34]$ & $\mathrm{X}$ & & & & & & & \\
\hline$[35]$ & $\mathrm{X}$ & & & & & & & \\
\hline$[54]$ & & & & & & & $\mathrm{X}$ & \\
\hline$[82]$ & & & & & $\mathrm{X}$ & & & \\
\hline$[83]$ & & & & & & $\mathrm{X}$ & & \\
\hline
\end{tabular}

CR (Convergence requirements); CH(Challenges); PS(Privacy and security); $\operatorname{Tr}$ (Trends); SDP(Systems development and prototypes); IA(Implementation and adoption); ED(Education);

$S C$ (Standardization and compliance)

\subsection{Convergence Model for Patient Data}

We synthesize the identified themes into a multilayer model to characterize the research maturity by positioning existing work at different layers. This 5layer model (Figure 5) offers guidelines in identifying research opportunities, similar to the Schein's model of organizational culture $[84,85]$ where a subsequent layer builds on the previous layers(s). Layer 1 includes the vision, challenges, and requirements. The next step is implementation and development included in Layer 2, followed by system testing and adoption in Layer 3 . Layer 4 focuses on decision making, and the health outcomes are described and studied in Layer 5.

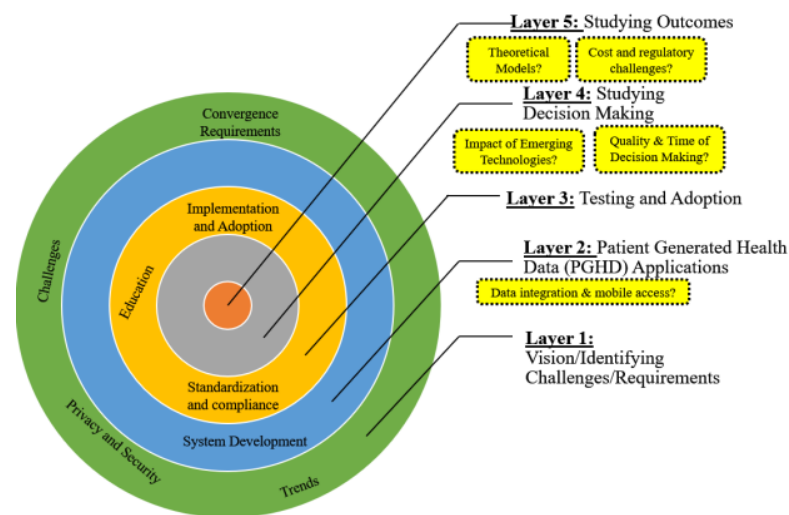

Figure 5. Convergence Model for Patient Data (CMPD)

Developed Using Schein's Model [84, 85]

We mapped the eight themes (Figure 5) into the CMPD layers. Using hermeneutics, convergence requirements $\{6\}$, challenges $\{4\}$, privacy and security $\{4\}$, and trends $\{5\}$ were mapped to Layer 1 (19 articles). Systems development and prototype $\{11\}$ were mapped to Layer 2 (11 articles). Implementation and adoption $\{13\}$, education $\{5\}$, and standardization
$\{5\}$ were mapped to Layer 3 (21 articles). Thus, all 53 articles were mapped into the first three CMPD layers. We find research gaps in decision making (Layer 4) and healthcare outcomes (Layer 5) in the converged environment (shown as dotted rectangles).

\section{A framework for decision making}

We next present the Quality and Timeliness (Q\&T) framework, based on the literature, using (a) scenarios of decision making and requirements and (b) implementation for the converged environment.

\subsection{Scenarios for decision making}

An intriguing dichotomy exists in healthcare where some healthcare decisions focus on high quality without time constraints, while other decisions are time critical even if the quality of information is still uncertain and evolving such as in disease/epidemic tracking. However, interestingly there can be situations where decisions may need both high quality and timeliness such as emergency response systems. Below, we discuss numerous scenarios of the Q\&T framework (Figure 6) where time sensitivity increases from green to red.

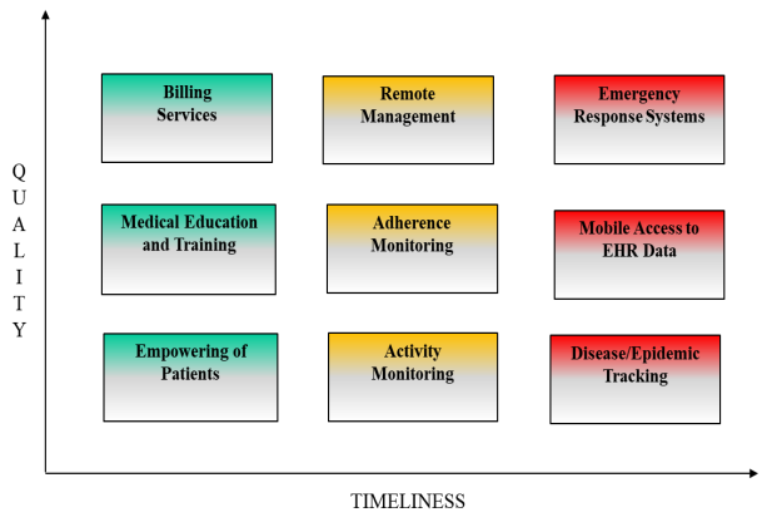

Figure 6. A classification of several PGHD and HPGD applications

Timely availability of patient data from both PGHD and HPGD is becoming crucial for patient care. Monitoring of patients through PGHD applications can enhance the quality of care and save lives potentially. With availability of wide range of sensors (such as ECG, SpO2), the PGHD can enhance the timeliness and quality of care [86]. While huge amount of data is generated for a patient contributing to the Big Data revolution, timeliness of data availability still remains a challenge in the healthcare context [87]. Timeliness and quality requirements are presented in Figure $6 \&$ more details in Table 2 for the considered scenarios. 
Table 2. Quality and Timeliness Requirements

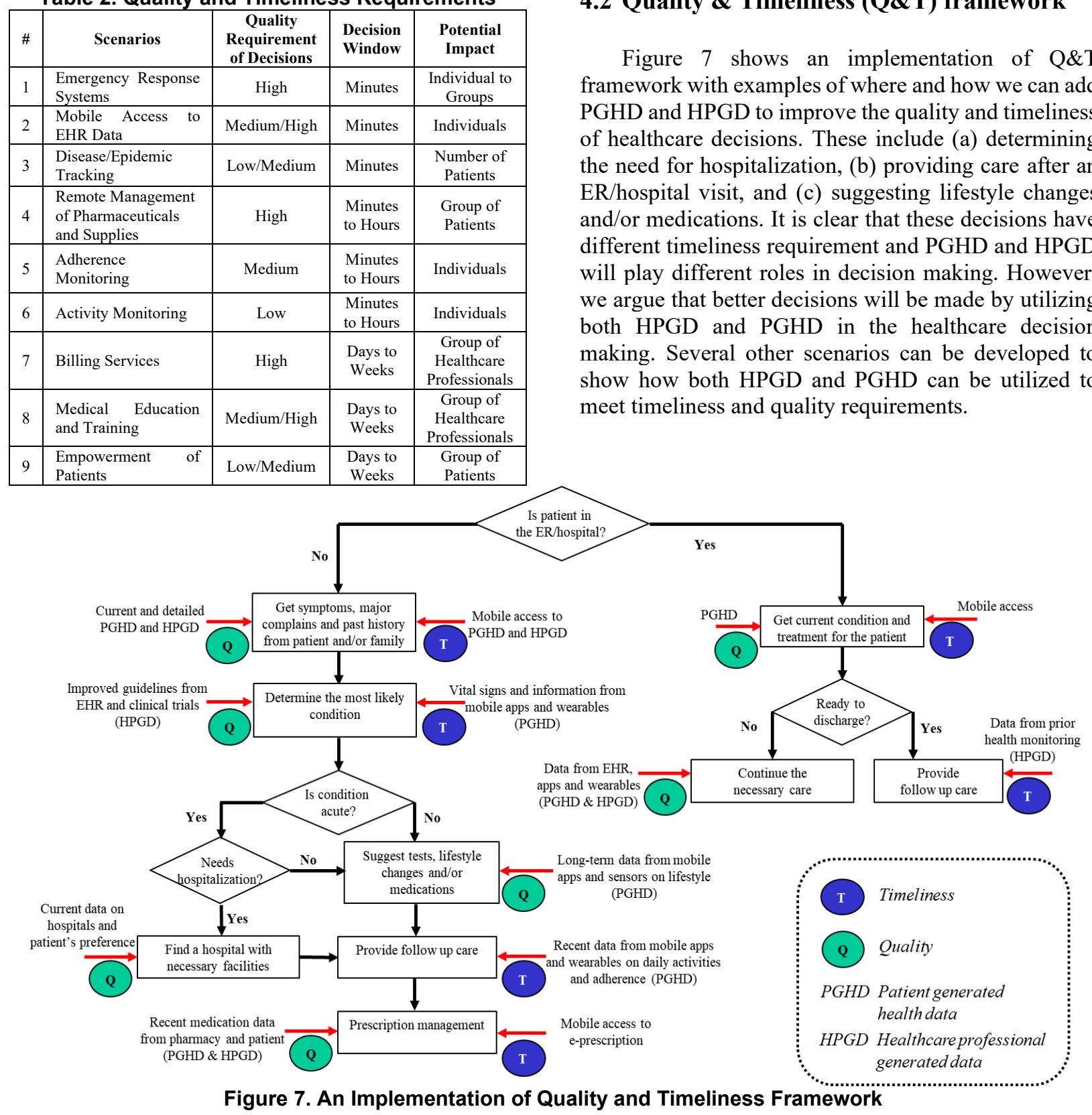

\subsection{Quality \& Timeliness (Q\&T) framework}

\section{\&T} 4

.

.

.

.

\section{Discussion}

The convergence of PGHD and HPGD offers improved healthcare decision making and effective and personalized interventions [78]. One of the major challenges is to deliver timely and context-sensitive PGHD to the decision makers in their location at the best possible quality and lowest cost.

\subsection{Recommendations for future research}

.

.

.

.


to have access to PGHD and HPGD from anywhere, anytime. Several authors [77, 82] and systems [21, 49] address parts of the challenge, but an integrated approach is required to address challenges related to mobile devices (display, battery, and network access), access to multiple mobile networks (connectivity), and end-to-end security (trusted networks).

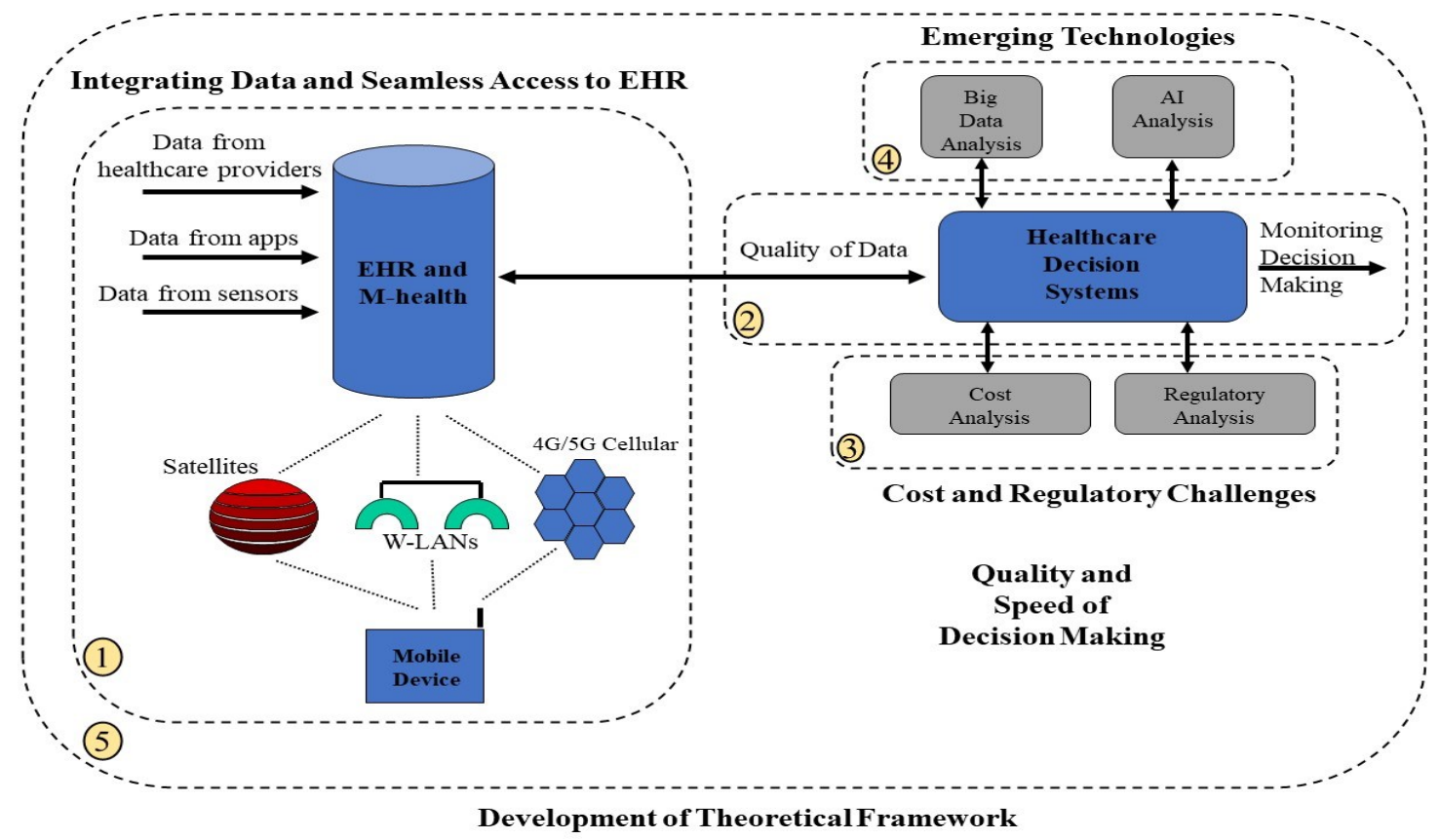

Figure 8. Visualization of Future Research Directions

5.1.2 Quality and speed of decision making. None of the articles addressed the quality of decision making. However, the speed of decision making was included in certain articles. Even with a lack of metrics, one way to study the "quality" of decision making is to focus on how and where converged information may be used in the decision making process. Decisions can then be compared with historical data to see improvements in healthcare quality. We presented high-level details of Q\&T Framework for subsequent implementation and evaluation. Multiple metrics, including the speed of decision making, the number of errors in decision making, patient outcomes, and healthcare costs, can be utilized. The comparison can also be done both immediately after the converged environment becomes available and longitudinally over time. There is a need for protocols to deal with data quality challenges for PGHD (incorrect wearable data).

5.1.3 Cost items and regulatory challenges. The cost items for the convergence are: (1) the cost of HPGD application, (2) the cost of integrating information from multiple sources, (3) the cost of PGHD applications, (4) the network and device cost, and (5) the management, regulatory, and maintenance cost. Therefore, a research study could evaluate the cost effectiveness using the return on investment (ROI) metric. Work is needed to address the liabilities related to errors and their propagation through PGHD applications [88] and wearables. It is necessary to study regulatory cost if the FDA treats PGHD applications as a medical device. More clarity on the emerging regulatory environment will be beneficial as the integrated environment is implemented. Globally, the impact of countries' individual regulations on data privacy and security, especially for patient health data, should be addressed in future research.

5.1.4 Impact of emerging technologies. PGHD applications generate data as they monitor activities and conditions continuously. The amount of data in HPGD applications also increases with time. Decision making will involve analysis of a vast amount of data, which includes controlled and uncontrolled data from HPGD and PGHD applications respectively in multiple forms (structured and unstructured). Examining for short-term and long-term patterns using artificial intelligence [89] can facilitate personalized decision making using smart systems [90]. Future research can study the impact of emerging technologies on HP in terms of (a) the need for additional skills and (b) workload for decision making. 5.1.5 Development of a theoretical framework. We did not find any studies using theories for decision making or developing theoretical framework. To add rigor, a theoretical framework is necessary. Several 
interesting phenomena include patient empowerment, comfort with PGHD applications, data quality and security challenges, and difficulties in accessing HPGD applications while being mobile. Research can use the lenses of the Health Promotion Model, the Humanistic Nursing Theory, the Cognitive Load Theory, the Social Cognitive Theory, the Theory of Planned Action and Behavior, to explain these phenomena and evaluation using field studies.

\subsection{Limitations of the study}

Our search included literature published in English language only, and thus insights from the literature in other languages are not included in this review. Research in PGHD applications and wearables is quite dynamic and therefore some observations may change over time. Finally, additional research should address how to improve the quality of data provided by the patient to improve decision making by addressing motivation to deliver PGHD and perceived usefulness.

\section{Conclusion}

Decision making for healthcare has been an important area of research due to both its complexity and the potential impact of decisions on patients. This review generates several interesting insights. Eight themes were identified: convergence requirements, challenges, privacy and security, trends, systems development and prototypes, implementation and adoption, education, and standardization and compliance. Besides answering our first research question, these themes helped us in proposing the Convergence Model for Patient Data (CMPD). This model depicts the maturity of research in layers such that it can be used to evaluate and categorize current and future research on convergence of PGHD and HPGD. A framework is presented to identify quality and timeliness requirements and to provide necessary decision support for scenarios where timeliness is more important than accuracy.

Decision making could involve additional data from PGHD applications and wearables in designing personalized interventions. The interventions can be further improved by using current clinical guidelines. Using monitored data, appropriate decisions can be made quickly to improve health outcomes. Healthcare professionals may use PGHD, for example, to determine the type and frequency of dosing and use lifestyle data to determine personalized wellness interventions. This helps in addressing our second research question. We hope that the findings of this paper should encourage researchers to focus on the recommendations for future research, including changes in the quality of decision making due to PGHD and its effect on healthcare outcomes. One way to achieve integration could involve promoting EHR vendors to also provide m-health apps to address both interoperability and trust.

\section{References}

1.N. Meskens and A. Guinet, "Decision making in healthcare," Decision Support Systems, vol. 55, no. 2, 2013.

2.H.K. Bhargava and A.N. Mishra, "Electronic medical records and physician productivity: Evidence from panel data analysis," Management Science, vol. 60, no. 10, 2014, pp. 2543-2562.

3. A. Bourouis, et al., "An intelligent mobile based decision support system for retinal disease diagnosis," Decision Support Systems, vol. 59, 2014, pp. 341-350.

4. M. Cho, et al., "Evaluating the effect of best practices for business process redesign: An evidence-based approach based on process mining techniques," Decision Support Systems, vol. 104, 2017, pp. 92-103.

5.N. Huba and Y. Zhang, "Designing patient-centered personal health records (PHRs): health care professionals' perspective on patient-generated data," Journal of medical systems, vol. 36, no. 6, 2012, pp. 3893-3905.

6. D.C. Lavallee, et al., " $m$ Health and patient generated health data: stakeholder perspectives on opportunities and barriers for transforming healthcare," Mhealth, vol. 6, 2020.

7. R. Sayeed, et al., "SMART Markers: collecting patient-generated health data as a standardized property of health information technology," NPJ digital medicine, vol. 3, no. 1, 2020, pp. 1-8.

8. N. Singh and U. Varshney, "Medication adherence: A method for designing context-aware reminders," International journal of medical informatics, vol. 132, 2019, pp. 103980.

9. N. Singh and U. Varshney, "IT-based reminders for medication adherence: systematic review, taxonomy, framework and research directions," European Journal of Information Systems, vol. 29, no. 1, 2020, pp. 84-108.

10. C.M. DesRoches, et al., "Electronic health records' limited successes suggest more targeted uses," Health affairs, vol. 29, no. 4, 2010, pp. 639-646.

11. R. Kohli and S.S.-L. Tan, "Electronic health records: how can IS researchers contribute to transforming healthcare?," Mis Quarterly, vol. 40, no. 3, 2016, pp. 553-573.

12. U. Varshney, "Pervasive Healthcare Computing," Pervasive Healthcare Computing: EMR/EHR, Wireless and Health Monitoring, ISBN 978-1-4419-0214-6. Springer-Verlag US, 2009, 2009.

13. L. Wu, et al., "The adoption of mobile healthcare by hospital's professionals: An integrative perspective," Decision Support Systems, vol. 51, no. 3, 2011, pp. 587-596.

14. U. Varshney, "Mobile health: Four emerging themes of research," Decision Support Systems, vol. 66, 2014, pp. 20-35.

15. D. Romanow, et al., "Editor's comments: riding the wave: past trends and future directions for health IT research," MIS quarterly, 2012, pp. iii-x.

16. S. El-Sappagh, et al., "A mobile health monitoring-andtreatment system based on integration of the SSN sensor ontology and the HL7 FHIR standard," BMC medical informatics and decision making, vol. 19, no. 1, 2019, pp. 97.

17. K.D. Mandl, et al., "The SMART Platform: early experience enabling substitutable applications for electronic health records," $J$ Am Med Inform Assoc, vol. 19, no. 4, 2012, pp. 597-603; DOI 10.1136/amiajnl-2011-000622.

18. J.L. Warner, et al., "SMART precision cancer medicine: a FHIR-based app to provide genomic information at the point of care," J Am Med Inform Assoc, vol. 23, no. 4, 2016, pp. 701-710; DOI 10.1093/jamia/ocw015. 
19. M. Khalemsky and D.G. Schwartz, "Emergency response community effectiveness: A simulation modeler for comparing emergency medical services with smartphone-based samaritan response," Decision Support Systems, vol. 102, 2017, pp. 57-68. 20. C. Keyworth, et al., "What maximizes the effectiveness and implementation of technology-based interventions to support healthcare professional practice? A systematic literature review," BMC Medical Informatics and Decision Making, vol. 18, no. 1, 2018, pp. 93; DOI 10.1186/s12911-018-0661-3.

21. J.C. Mandel, et al., "SMART on FHIR: a standards-based, interoperable apps platform for electronic health records," Journal of the American Medical Informatics Association, vol. 23, no. 5, 2016, pp. 899-908; DOI 10.1093/jamia/ocv189.

22. S.J. Iribarren, et al., "Scoping review and evaluation of SMS/text messaging platforms for mHealth projects or clinical interventions," International journal of medical informatics, vol. 101, 2017, pp. 28-40.

23. L. Liu, et al., "Smart homes and home health monitoring technologies for older adults: A systematic review," International journal of medical informatics, vol. 91, 2016, pp. 44-59.

24. Z. Yin, et al., "A systematic literature review of machine learning in online personal health data," Journal of the American Medical Informatics Association, vol. 26, no. 6, 2019, pp. 561-576; DOI 10.1093/jamia/ocz009.

25. A. Liberati, et al., "The PRISMA Statement for Reporting Systematic Reviews and Meta-Analyses of Studies That Evaluate Health Care Interventions: Explanation and Elaboration," Annals of Internal Medicine, vol. 151, no. 4, 2009, pp. W-65-W-94.

26. D. Moher, et al., "Preferred reporting items for systematic review and meta-analysis protocols (PRISMA-P) 2015 statement," Systematic reviews, vol. 4, no. 1, 2015, pp. 1.

27. J.V. Carvalho, et al., "A Maturity model for hospital information systems," Journal of Business Research, vol. 94, 2019 , pp. 388-399.

28. U. Varshney, "A model for improving quality of decisions in mobile health," Decision Support Systems, vol. 62, 2014, pp. 66-77. 29. H. Snyder, "Literature review as a research methodology: An overview and guidelines," Journal of Business Research, vol. 104, 2019, pp. 333-339.

30. AHRQ, "Automated-Entry Patient Generated Health Data for Chronic Conditions: The Evidence on Health Outcomes," 2019; https://effectivehealthcare.ahrq.gov/products/health-datamapping/protocol.

31. M.J. Reading and J.A. Merrill, "Converging and diverging needs between patients and providers who are collecting and using patient-generated health data: an integrative review," Journal of the American Medical Informatics Association, vol. 25, no. 6, 2018, pp. 759-771.

32. M.C. Lacity and M.A. Janson, "Understanding qualitative data: A framework of text analysis methods," Journal of Management Information Systems, vol. 11, no. 2, 1994, pp. 137-155.

33. M.B. Miles, et al., Qualitative Data Analysis: A Methods Sourcebook, SAGE Publications, 2018.

34. F. Fritz, et al., "CIS-based registration of quality of life in a single source approach," BMC Medical Informatics \& Decision Making, vol. 11, no. 1, 2011, pp. 26-34; DOI 10.1186/1472-694711-26.

35. D. Lorence, et al., "Transaction-Neutral Implanted Data Collection Interface as EMR Driver: A Model for Emerging Distributed Medical Technologies," Journal of Medical Systems, vol. 34, no. 4, 2010, pp. 609-617; DOI 10.1007/s10916-009-92749.

36. J. Parkinson, "Getting real in clinical trials," Nature Reviews Drug Discovery, vol. 13, no. 9, 2014, pp. 639-640; DOI 10.1038/nrd4415.

37. B. Le Grice, "Transforming Health Care: The connected ecosystem and digital health," Australasian Biotechnology, vol. 27, no. 1, 2017, pp. 32-33.
38. B.K. Wiederhold, "Using Your Digital Phenotype to Improve Your Mental Health," Book Using Your Digital Phenotype to Improve Your Mental Health, Series Using Your Digital Phenotype to Improve Your Mental Health 19, ed., Editor ed.^eds., Mary Ann Liebert, Inc., 2016, pp. 419-419.

39. C. Manz, et al., "Marketing to physicians in a digital world," The New England Journal of Medicine, vol. 371, no. 20, 2014, pp. 1857-1859; DOI 10.1056/NEJMp1408974.

40. P.F. Cipriano, "President's Perspective. Technology in transition," American Nurse, vol. 46, no. 5, 2014, pp. 3-3.

41. M. Dasari, et al., "Implementation of a Hospital Electronic Surgical Registry in a Lower-Middle-Income Country," World Journal of Surgery, vol. 40, no. 12, 2016, pp. 2840-2846; DOI 10.1007/s00268-016-3654-3.

42. S.C. Denaxas, et al., "The tip of the iceberg: challenges of accessing hospital electronic health record data for biological data mining," Book The tip of the iceberg: challenges of accessing hospital electronic health record data for biological data mining, Series The tip of the iceberg: challenges of accessing hospital electronic health record data for biological data mining 9, ed., Editor ed.^eds., BioMed Central, 2016, pp. 1-4.

43. L.A. Gallagher, "Accessing and sharing data to avoid security risks," Nurse Practitioner, vol. 38, no. 5, 2013, pp. 8-11; DOI 10.1097/01.NPR.0000428822.80127.6a.

44. M. Bajwa, "mHealth Security," Pakistan Journal of Medical Sciences, vol. 30, no. 4, 2014, pp. 904-907; DOI 10.12669/pjms.304.5210.

45. C.J. Wang and D.J. Huang, "The HIPAA conundrum in the era of mobile health and communications," JAMA: Journal of the American Medical Association, vol. 310, no. 11, 2013, pp. 11211122; DOI 10.1001/jama.2013.219869.

46. M. Bajwa, "Emerging 21st Century Medical Technologies," Pakistan Journal of Medical Sciences, vol. 30, no. 3, 2014, pp. 649655; DOI 10.12669/pjms.303.5211.

47. B.I. Fox and B.G. Felkey, "Pharmacy Automation and Technology - Two Important Topics for 2012: Electronic Health Records and mHealth," Hospital Pharmacy, vol. 47, no. 1, 2012, pp. 62-63.

48. A.B. McCoy, et al., "State of the art in clinical informatics: evidence and examples," Yearbook Of Medical Informatics, vol. 8, 2013, pp. 13-19.

49. R.A. Bloomfield, et al., "Opening the Duke electronic health record to apps: Implementing SMART on FHIR," International Journal of Medical Informatics, vol. 99, 2017, pp. 1-10; DOI https://doi.org/10.1016/j.ijmedinf.2016.12.005.

50. F.C. Day, et al., "Feasibility study of an EHR-integrated mobile shared decision making application," International Journal of Medical Informatics, vol. 124, 2019, pp. 24-30; DOI https://doi.org/10.1016/j.ijmedinf.2019.01.008.

51. D.A. Alegría, et al., "Using tablets to support self-regulated learning in a longitudinal integrated clerkship," Medical Education Online, vol. 19, no. 1, 2014, pp. 23638; DOI 10.3402/meo.v19.23638.

52. M.A. Nuss, et al., "Real-time use of the iPad by third-year medical students for clinical decision support and learning: a mixed methods study," Journal of Community Hospital Internal Medicine Perspectives (JCHIMP), vol. 4, no. 4, 2014, pp. 1-7; DOI 10.3402/jchimp.v4.25184.

53. T.W. Toh, et al., "Development of a virtual patient record mobile app for pharmacy practice education," Archives of Pharmacy Practice, vol. 5, no. 2, 2014, pp. 66-71; DOI 10.4103/2045080X.132650.

54. J.S. Luo, "Informatics Competencies for the Clinical Practice: Medical Information," Primary Psychiatry, vol. 16, no. 8, 2009, pp. 22-24.

55. M. Sharp and D. O'Sullivan, "Mobile Medical Apps and mHealth Devices: A Framework to Build Medical Apps and mHealth Devices in an Ethical Manner to Promote Safer Use - A 
Literature Review," Studies In Health Technology And Informatics, vol. 235, 2017, pp. 363-367.

56. J.H. Song, et al., "Preliminary comparison of mHealth architectures based on MU2 criteria," Studies In Health Technology And Informatics, vol. 192, 2013, pp. 1127-1127.

57. P. Plastiras and D. O'Sullivan, "Exchanging personal health data with electronic health records: A standardized information model for patient generated health data and observations of daily living," International Journal of Medical Informatics, vol. 120, 2018, pp. 116-125; https://doi.org/10.1016/j.ijmedinf.2018.10.006.

58. F.A. Drews, et al., "Electronic health record on the go: Device form factor and Fitts' law," International Journal of Medical Informatics, vol. 111, 2018, pp. 37-44; DOI https://doi.org/10.1016/j.ijmedinf.2017.12.010.

59. K. Lee, et al., "Effect of self-monitoring on long-term patient engagement with mobile health applications," PLOS ONE, vol. 13, no. 7, 2018, pp. 1-12; DOI 10.1371/journal.pone.0201166.

60. J.J. Saleem, et al., "Investigating the need for clinicians to use tablet computers with a newly envisioned electronic health record," International Journal of Medical Informatics, vol. 110, 2018, pp. 25-30; DOI https://doi.org/10.1016/j.ijmedinf.2017.11.013.

61. Z. Cai, et al., "Towards secure and flexible EHR sharing in mobile health cloud under static assumptions," Cluster Computing, vol. 20, no. 3, 2017, pp. 2415-2422; DOI 10.1007/s10586-0170796-5.

62. U. Guo, et al., "Electronic health record innovations: Helping physicians -- One less click at a time," Health Information Management Journal, vol. 46, no. 3, 2017, pp. 140-144; DOI $10.1177 / 1833358316689481$.

63. A. Motulsky, et al., "Using mobile devices for inpatient rounding and handoffs: an innovative application developed and rapidly adopted by clinicians in a pediatric hospital," Journal of the American Medical Informatics Association, vol. 24, no. e1, 2017, pp. e69-e78; DOI 10.1093/jamia/ocw107.

64. M. Peleg, et al., "Assessment of a personalized and distributed patient guidance system," International Journal of Medical Informatics, vol. 101, 2017, pp. 108-130; DOI https://doi.org/10.1016/j.ijmedinf.2017.02.010.

65. S. Biddle, "Nursing informatics. The intersection of policy and informatics," Nursing Management, vol. 47, no. 2, 2016, pp. 12-13; DOI 10.1097/01.NUMA.0000479453.73651.89.

66. B. Schooley, et al., "Impacts of mobile tablet computing on provider productivity, communications, and the process of care," International Journal of Medical Informatics, vol. 88, 2016, pp. 6270; DOI 10.1016/j.ijmedinf.2016.01.010.

67. N. Skyttberg, et al., "How to improve vital sign data quality for use in clinical decision support systems? A qualitative study in nine Swedish emergency departments," BMC Medical Informatics \& Decision Making, vol. 16, 2016, pp. 1-12; DOI 10.1186/s12911016-0305-4.

68. D.M. Zive, et al., "Implementation of a Novel Electronic Health Record-Embedded Physician Orders for Life-Sustaining Treatment System," Journal Of Medical Systems, vol. 40, no. 11, 2016, pp. 245-245.

69. M. Bąkała and M. Michalski, "Mobile Applications as a Tool Supporting Healthcare Management," Polish Journal of Management Studies, vol. 12, no. 1, 2015, pp. 7-15.

70. W. Choi, et al., "Early Experiences with Mobile Electronic Health Records Application in a Tertiary Hospital in Korea," Healthcare Informatics Research, vol. 21, no. 4, 2015, pp. 292-298; DOI 10.4258/hir.2015.21.4.292.

71. A. Landman, et al., "A mobile app for securely capturing and transferring clinical images to the electronic health record: description and preliminary usability study," JMIR Mhealth And Uhealth, vol. 3, no. 1, 2015, pp. e1-e1; DOI 10.2196/mhealth.3481. 72. S. Mantwill, et al., "EMPOWER-support of patient empowerment by an intelligent self-management pathway for patients: study protocol," BMC Medical Informatics \& Decision Making, vol. 15, no. 1, 2015, pp. 1-7; DOI 10.1186/s12911-0150142-x.

73. S. Marceglia, et al., "A Standards-Based Architecture Proposal for Integrating Patient mHealth Apps to Electronic Health Record Systems," Applied Clinical Informatics, vol. 6, no. 3, 2015, pp. 488505; DOI 10.4338/ACI-2014-12-RA-0115.

74. K. Ho, "Key Trends in eHealth to Propel Health Transformation and Education," UBC Medical Journal, vol. 5, no. 2, 2014, pp. 6-9. 75. G. Mehl and A. Labrique, "Prioritizing integrated mHealth strategies for universal health coverage," Science, vol. 345, no. 6202, 2014, pp. 1284-1287; DOI 10.1126/science.1258926.

76. R. Wuerth, et al., "Top 10 tips for effective use of electronic health records," Pediatrics \& Child Health (1205-7088), vol. 19, no. 3, 2014, pp. 138-138.

77. W. Choi, et al., "Development of mobile electronic health records application in a secondary general hospital in Korea," Healthcare Informatics Research, vol. 19, no. 4, 2013, pp. 307-313; DOI 10.4258/hir.2013.19.4.307.

78. J.Y. Kim, et al., "Needs analysis and development of a tailored mobile message program linked with electronic health records for weight reduction," International Journal of Medical Informatics, vol. 82, no. 11, 2013, pp. 1123-1132; DOI https://doi.org/10.1016/j.ijmedinf.2013.08.004.

79. P.C. Tang, et al., "Online disease management of diabetes: Engaging and Motivating Patients Online With Enhanced Resources-Diabetes (EMPOWER-D), a randomized controlled trial," Journal of the American Medical Informatics Association, vol. 20, no. 3, 2013, pp. 526-534; DOI 10.1136/amiajnl-2012001263 .

80. F. Fritz, et al., "Qualitative and quantitative evaluation of EHRintegrated mobile patient questionnaires regarding usability and cost-efficiency," International Journal of Medical Informatics, vol. 81, no. 5, 2012, pp. 303-313; DOI https://doi.org/10.1016/j.ijmedinf.2011.12.008.

81. V. Camlek, "Healthcare mobile information flow," Information Services \& Use, vol. 31, no. 1-2, 2011, pp. 23-30.

82. D. O'Sullivan, et al., "Mobile case-based decision support for intelligent patient knowledge management," Health Informatics Journal, vol. 13, no. 3, 2007, pp. 179-193; DOI $10.1177 / 1460458207079839$.

83. I. Cricelli, "Use of personal digital assistant devices in order to access, consult and apply a corpus of clinical guidelines and decision-based support documentation like the Italian SPREAD Guidelines on stroke disease," Neurological Sciences: Official Journal of the Italian Neurological Society and of the Italian Society of Clinical Neurophysiology, vol. 27 Suppl 3, 2006, pp. S238-S239. 84. E.H. Schein, "Organizational culture and leadership JosseyBass," San Francisco, CA, 1992.

85. E.H. Schein, Organizational culture and leadership, John Wiley \& Sons, 2010.

86. R. Chakravorty, "A programmable service architecture for mobile medical care," Proc. Fourth Annual IEEE International Conference on Pervasive Computing and Communications Workshops (PERCOMW'06), IEEE, 2006, pp. 5 pp.-536.

87. W. Liu and E. Park, "Big data as an e-health service," Proc. 2014 International Conference on Computing, Networking and Communications (ICNC), IEEE, 2014, pp. 982-988.

88. S. Sneha and U. Varshney, "A framework for enabling patient monitoring via mobile ad hoc network," Decision Support Systems, vol. 55, no. 1, 2013, pp. 218-234.

89. K.-H. Yu, et al., "Artificial intelligence in healthcare," Nature Biomedical Engineering, vol. 2, no. 10, 2018, pp. 719.

90. I. Martin-Loeches, et al., "Intensive care medicine in 2050: nanotechnology. Emerging technologies and approaches and their impact on critical care," Intensive Care Medicine, vol. 44, no. 8, 2018, pp. 1299-1301. 